\title{
Optimizing Data Recording for the NIF Core Diagnostic X-ray Streak Camera
}

D.H. Kalantar, P.M. Bell, T.S. Perry, N. Sewall, C. Diamond, and K. Piston

This article was submitted to The $13^{\text {th }}$ Topical Conference on High-Temperature Diagnostics, Tuscon, AZ, June $18-20,2000$

\section{June 13, 2000}

U.S. Department of Energy

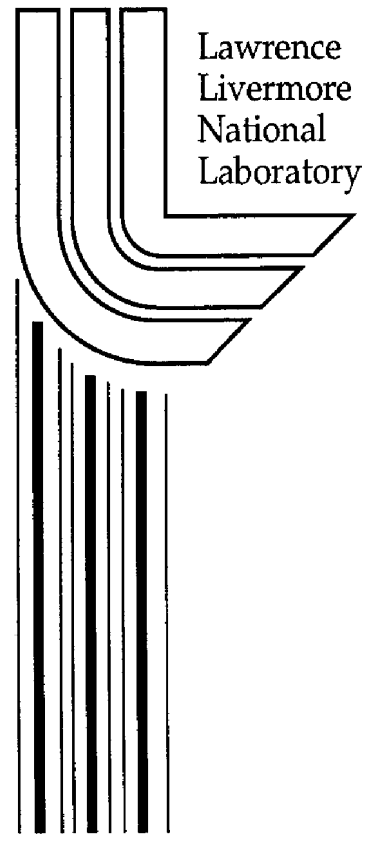




\section{DISCLAIMER}

This document was prepared as an account of work sponsored by an agency of the United States Government. Neither the United States Government nor the University of California nor any of their employees, makes any warranty, express or implied, or assumes any legal liability or responsibility for the accuracy, completeness, or usefulness of any information, apparatus, product, or process disclosed, or represents that its use would not infringe privately owned rights. Reference herein to any specific commercial product, process, or service by trade name, trademark, manufacturer, or otherwise, does not necessarily constitute or imply its endorsement, recommendation, or favoring by the United States Government or the University of California. The views and opinions of authors expressed herein do not necessarily state or reflect those of the United States Government or the University of California, and shall not be used for advertising or product endorsement purposes.

This is a preprint of a paper intended for publication in a journal or proceedings. Since changes may be made before publication, this preprint is made available with the understanding that it will not be cited or reproduced without the permission of the author.

This report has been reproduced directly from the best available copy.

Available electronically at http://www.doc.gov/bridge

Available for a processing fee to U.S. Department of Energy

And its contractors in paper from

U.S. Department of Energy

Office of Scientific and Technical Information

P.O. Box 62

Oak Ridge, TN 37831-0062

Telephone: (865) 576-8401

Facsimile: (865) 576-5728

E-mail: reports@adonis.osti.gov

Available for the sale to the public from

U.S. Department of Commerce

National Technical Information Service

5285 Port Royal Road

Springfield, VA 22161

Telephone: (800) 553-6847

Facsimile: (703) 605-6900

E-mail: orders@ntis.fedworld.gov

Online ordering: http:/ / www.ntis.gov/ordering.htm

\section{OR}

Lawrence Livermore National Laboratory

Technical Information Department's Digital Library

http: / / www.llnl.gov/tid/Library.html 
Optimizing data recording for the NIF core diagnostic $x$-ray streak camera D. H. Kalantar, P. M. Bell, T. S. Perry, N. Sewall, C. Diamond, K. Piston

Introduction

The $\mathrm{x}$-ray streak camera is an important instrument for recording a continuous time history of $x$-ray emitted from laser target experiments. X-ray streak cameras were used to diagnose experiments in inertially confined fusion and high energy density sciences on the Nova laser. These streak cameras are now used for similar experiments conducted at the OMEGA laser facility, and cameras of this type will be used for experiments on the National Ignition Facility (NIF).

The Nova $x$-ray streak cameras (SSCs) used a sealed optical image intensifier coupled to film to record the streaked $\mathrm{x}$-ray data. In order to develop the core $\mathrm{x}$-ray streak camera for NIF (ref Kimbrough) using a CCD based recording system, we evaluate the performance of the SSCs under a variety of detector configurations.

We performed laboratory bench characterization tests of the SSCs to measure the spatial resolution and to evaluate the dynamic range and signal to noise for different configurations of the SSC. We present results of these tests here.

\section{Bench characterization}

We made test exposures of the streak cameras on the bench using a multi-anode high voltage $\mathrm{x}$-ray source. A Ti anode was used to generate Ti K-alpha emission with an $x$-ray source voltage of $10 \mathrm{kV}$ and anode current ranging from 40-300 $\mu \mathrm{A}$. The SSCs were tested with different recording system configurations, as illustrated in Figure 1 and described in Table 1. In each case, the photocathode was identical, CsI coated onto a $1000 \AA$ Lexan substrate. A $12 \mu \mathrm{m}$ Ti filter was used to ensure that the x-rays incident on the photocathode were monochromatic $(4.5 \mathrm{keV})$.

Resolution test images were recorded with an array of 20-50 $\mu \mathrm{m}$ wide slits with a $1.5 \mathrm{~mm}$ separation placed in front of the $1 \mathrm{~mm}$ wide photocathode. Additional images of the fully illuminated $1 \mathrm{~mm}$ wide photocathode slit were recorded at a range of $\mathrm{x}$-ray source currents (x-ray fluences) and integration times. All test images were taken in the static mode, with the electron tube image positioned at the center of the screen. The gain 
of the optical image intensifier was set at medium, the gain of the direct electron microchannel plate was set with a bias voltage of $900 \mathrm{~V}$.

\section{FIGURE 1}

Component view of the $x$-ray streak camera

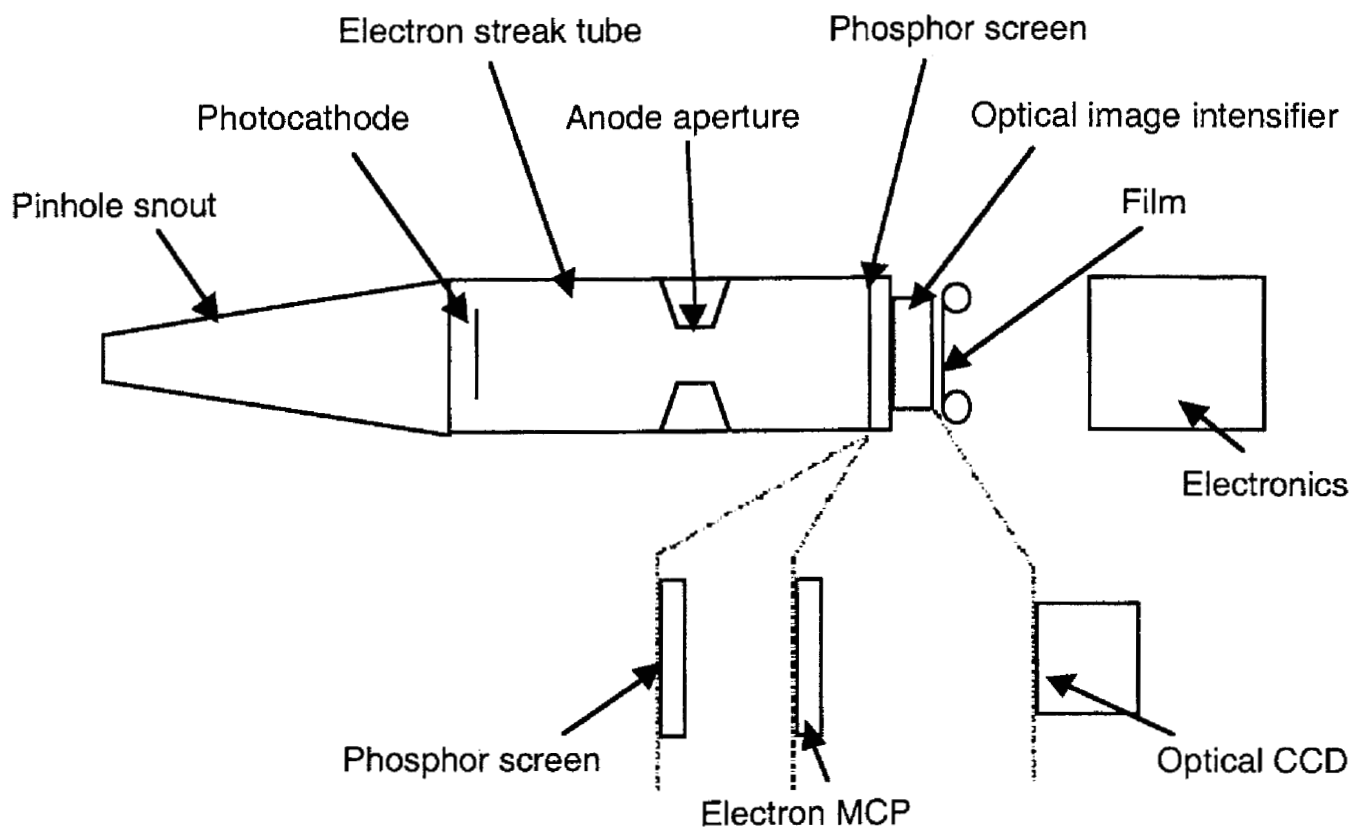

\section{TABLE 1}

Tests performed on the LLNL SSC streak cameras

\begin{tabular}{|c|c|c|c|c|c|}
\hline $\mathrm{SSC} 4$ & PC & electrons & $\mathrm{P} 20$ & $\mathrm{IIT} / \mathrm{P} 20$ & film \\
\hline SSC4 & PC & electrons & $\mathrm{P} 20$ & & film \\
\hline SSC1 & PC & electrons & e- MCP/P11 & & film \\
\hline $\mathrm{SSC} 4$ & PC & electrons & $\mathrm{P} 20$ & $\mathrm{ITT} / \mathrm{P} 20$ & $\mathrm{CCD}$ \\
\hline SSC4 & PC & electrons & $\mathrm{P} 20$ & & $\mathrm{CCD}$ \\
\hline SSC1 & PC & electrons & e- $M C P / P 11$ & & $\mathrm{CCD}$ \\
\hline
\end{tabular}




\section{Streak camera resolution}

The baseline configuration for the SSC includes an optical image intensifier with a P20 phosphor coupled to Kodak TMAX 3200 speed film. The resolution in this configuration was measured as a function of position along the photocathode. This is shown in Figure 1 as the full width at half maximum (fwhm) of the point spread function, calculated by deconvolving a lineout of the narrow slit image from the slit size itself. The resolution for this camera is best at the center of the photocathode and degrades towards the edges. The resolution is $X X$ at the center, and $X X 12 \mathrm{~mm}$ away from the center. Note that the degradation towards the edges is consistent with the design of the streak tube. It is cylindrically symmetric and it has a flat photocathode and phosphor screen. This resolution may in fact be adjusted for a compromise focus across a wider portion of the photocathode, but this was not done for these tests.

\section{FIGURE 2}

Plot of the resolution measurement... resolution as a function of position for SSC4 in the standard configuration... then comment on the values at $0,12 \mathrm{~mm}$ offset...

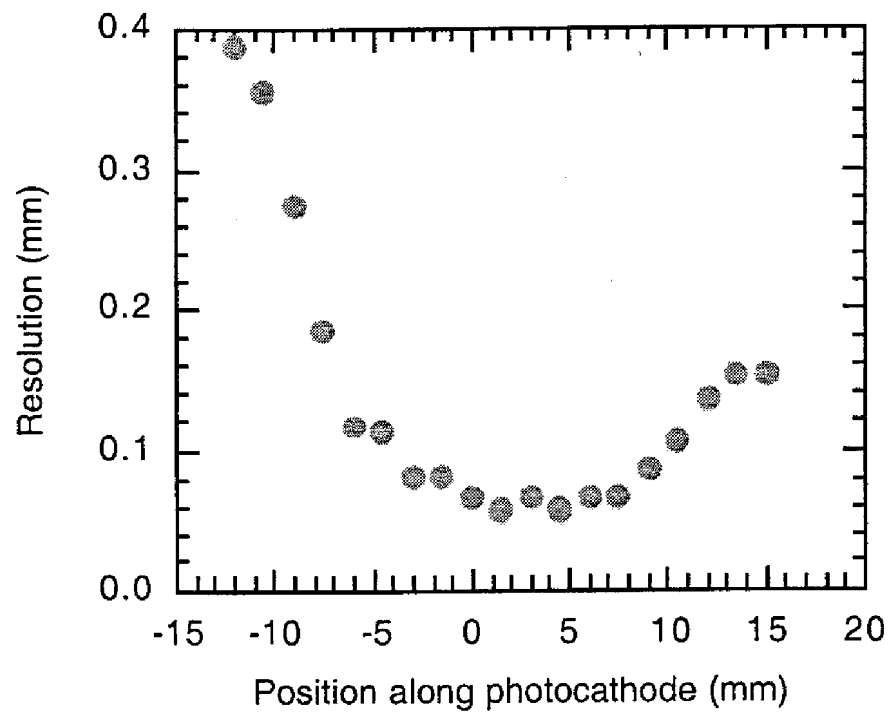

The test exposures were done in a static mode, and therefore do not include any dynamic saturation effects. We compared the spatial resolution measurements for the 
baseline SSC configuration with the other configurations listed in Table 1. These are discussed below. The resolution at the center of the photocathode and $\pm 12 \mathrm{~mm}$ offset are shown in Table 2 for each configuration. The resolution is improved by just removing the optical image intensifier, and also be replacing the electron sensitive phosphoroptical image intensifier combination with the direct electron sensitive microchannel plate. Note that in each case, the optical $4 \mathrm{kx} 4 \mathrm{k}$ CCD does not result in any significant degradation of the spatial resolution relative to film for any configuration tests either with single pixels or $2 \times 2$ binning. Using the CCD as the detector does start to impact the resolution if the binning is done as $4 \times 4$.

\section{TABLE 2}

Resolution (deconvolved) at $0,12 \mathrm{~mm}$ offset for all cases

Put in the table summarizing the results here.

Dynamic saturation of the electron optics

The resolution tests indicate that removing the optical image intensifier results in optimum performance. However, these bench tests do not include any dynamic saturation effects such as self focussing of the electron optics. In order to assess the viability of operating the camera in each configuration, we relate the static bench measurements to dynamics measurements.

We used the film calibration and exposure to establish the operation limits consistent with dynamic saturation such as self focussing of the electron beam at the anode aperture in the sweep tube. For each configuration, we recorded several images of the full photocathode area masked by a $1 \mathrm{~mm}$ wide slit as a function of $x$-ray exposure. The film density on film for the baseline SSC configuration was plotted against the integrated $x$-ray source output, defined as current times exposure time. This shows a linear response up to high exposures where the film is not well calibrated (Fig. 3).

Date recorded on Nova experiments that show saturation of the electron optics (pinching of the image) suggests that electron self focusing in the electron optics tube when the exposure on film is about $10-20 \mathrm{erg} / \mathrm{cm} 2$ at medium gain on the image intensifier. This was verified by comparing the exposure of another streak camera 
(SSCA) when it showed slight pinching. In this case, images of an array of slits showed time dependent effects as the electron beam was very close to the saturation level (Fig. 4). The average exposure was about $2.2 \mathrm{erg} / \mathrm{cm}$, corresponding to an x-ray source current-time exposure of $960 \mu \mathrm{A}$-s. Therefore, we establish this as the upper limit based on dynamic saturation of the electron optics. This is marked in Fgure 3, and all subsequent plots of SSC performance below. Note that the film exposure for SSC4 is about $10 \mathrm{erg} / \mathrm{cm} 2$ at this $x$-ray source flux, consistent with the typical experience on Nova at this gain setting. For high gain, the saturation level is $30 \mathrm{erg} / \mathrm{cm} 2$ or greater on the $5 \mathrm{~ns}$ sweep window (speed 1). For faster sweeps, the electron optic saturation level corresponds to less film exposure.

\section{FIGURE 3:}

Film exposure as a function of x-ray source flux obtained using SSC4 with an optical image intensifier set at medium gain.

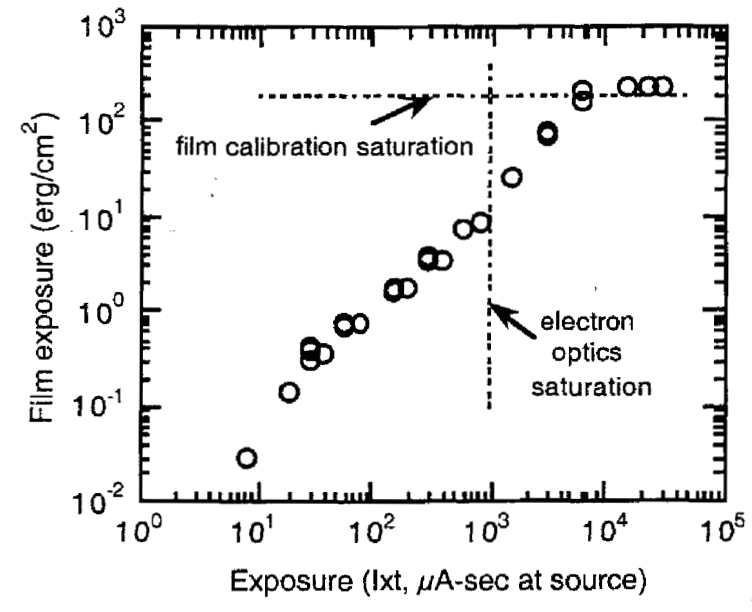

Visible light CCD

A Kodak $4 \mathrm{k} \times 4 \mathrm{k} C \mathrm{CD}$ is proposed as a replacement for film. This is a $36 \mathrm{~mm}$ square format CCD with 9 micron pixels. We used this to record the data for SSC4 in its otherwise standard configuration. The results are shown in Fig. 5. Here we observe CCD counts as a function of $x$-ray source output. The maximum CCD pixel count corresponds to an exposure of about $700 \mu \mathrm{A}-\mathrm{sec}$, which is close to the saturation level of 
960. The CCD has a high dynamic range. For low exposures, the pixels may be binned, but as indicated in Table 2 , the resolution is degraded with binning by more than $2 \times 2$.

\section{FIGURE 4:}

SSCA result showing slight pinching from an OMEGA shot.

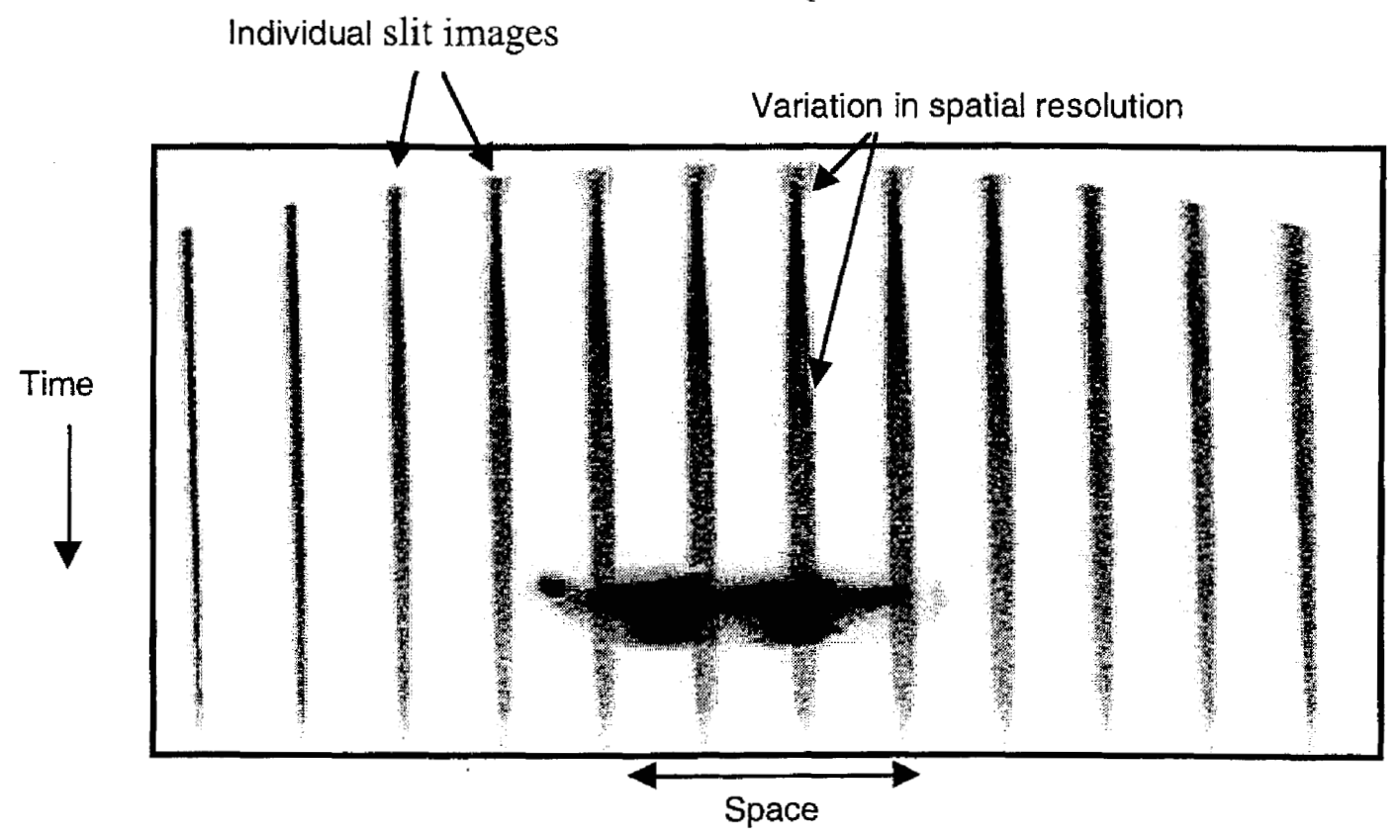

Optical image intensifier

Film and CCD images of the photocathode slit were recorded for SSC4 both with and without an optical image intensifier. A direct comparision of the results indicates that the image intensifier has an optical gain of about 3000 for the medium setting. As a result, the maximum exposure on film before electron beam saturation occurs is only about $0.003 \mathrm{erg} / \mathrm{cm} 2$, too low to be measured. The maximum CCD counts would be approximately 3 counts at the maximum $x$-ray exposure before dynamic electron optics saturation. This can be improved by binning, but at $2 \times 2$, this only increases the maximum CCD counts to approximately 12 . The bottom line is that the present cameras may not be used without an optical image intensifier. However, if the electron sensitive 
phosphor is improved or the optical CCD is significantly more sensitive, then this may be an option.

\section{FIGURE 5:}

Linearity for SSC4 with the optical CCD. Data is plotted for both cases with and without the IIT.

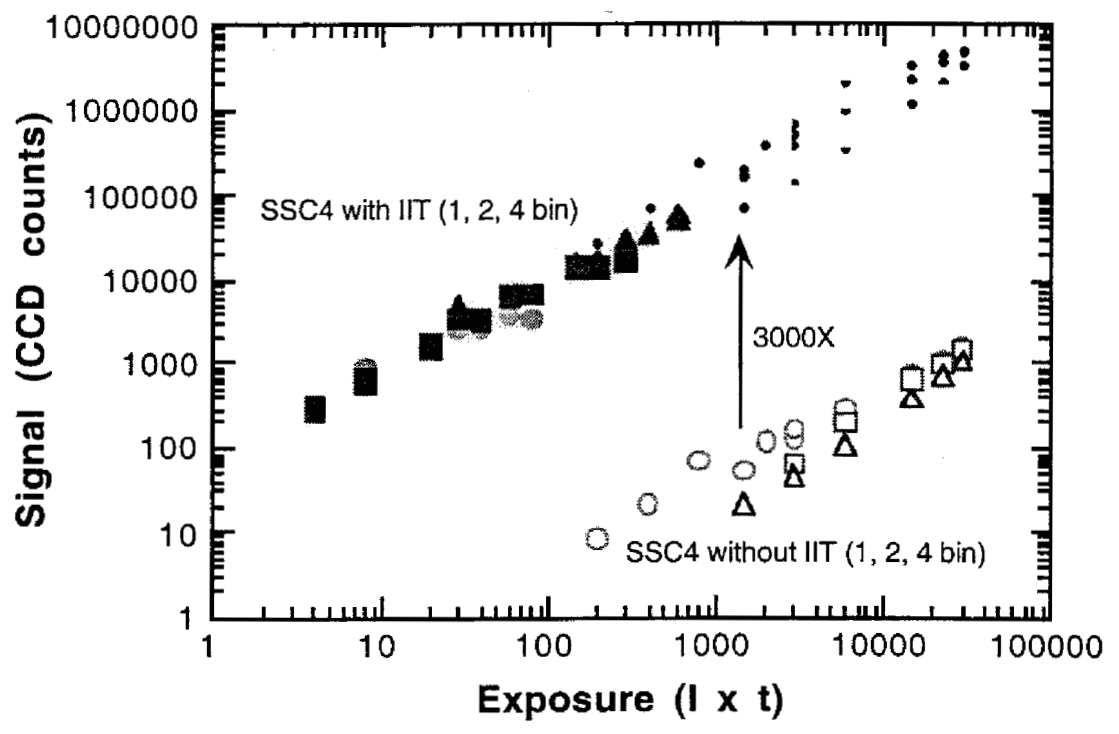

Microchannel plate

In one of the Nova SSCs, we replaced the electron sensitive phosphor and optical image intensifer with an electron sensitive microchannel plate. A complete comparison of the performance of this camera compared to the standard configuration is shown in reference XX. Results of the bench tests are shown in Fig. 6 . In the case where the data is recorded on film, the electron streak tube is expected to show saturation with a film exposure of only about $0.5 \mathrm{erg} / \mathrm{cm} 2$ (average over the full photocathode slit. The dynamic range is very low in this case since we run into severe noise about a factor of 10 lower than this.

In the case where the data is recorded using the $4 \mathrm{kx} 4 \mathrm{k} C \mathrm{CD}$, the electron optics tube saturates at about 1000 CCD counts. This allows a wide dynamic range even when the CCD pixels are binned with a $2 \times 2$ binning. Note, however, that the sensitivity of the CCD is reduced at the wavelegth of the P-11 phosphor used here. We may be able to improve this useable range by implementing a different phosphor output that is better matched to the CCD. 
FIGURE 6:

Linearity plot for SSC1 with the MCP. The data for both film and the $4 \mathrm{k} x 4 \mathrm{k}$ CCD are shown.

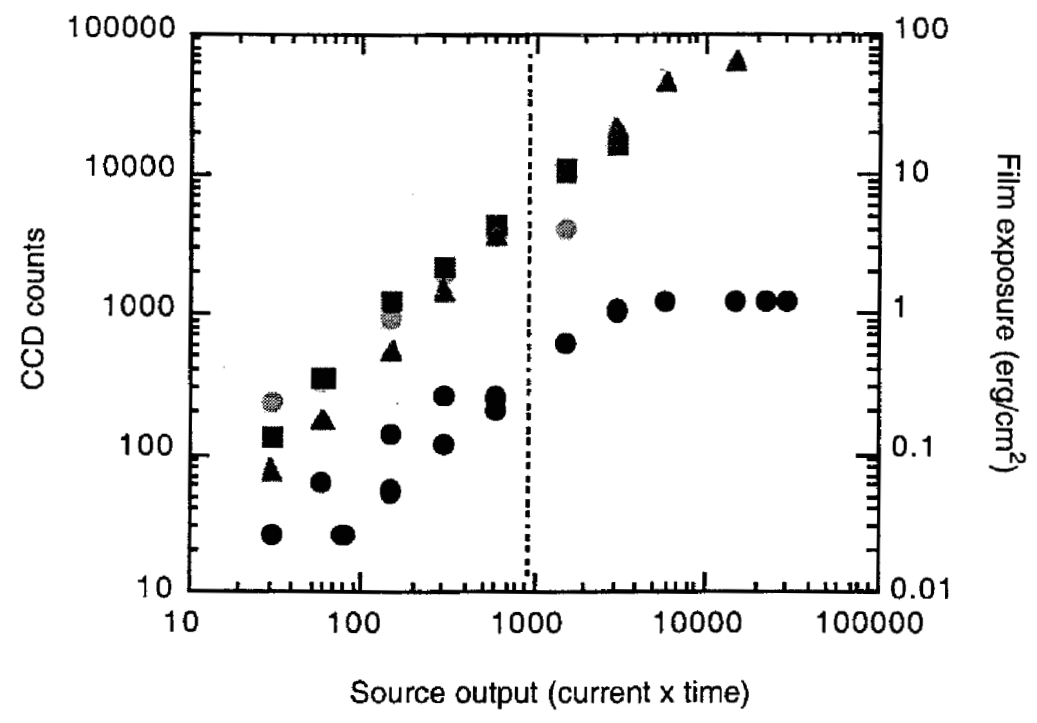

Comment on noise measurements - we need to have some discussion about the signal to noise analysis and results here.

\section{Conclusion}

The bench tests that were done provided a direct comparison of the resolution of the Nova SSCs under various configurations. This confirms that the optical image intensifier is a source of significant blurring. Removing this intensifier or replacing the phosphor-intensifier combination with an electron sensitive MCP improves the resolution significantly. Removing the image intensifier, however, results in signals that are too low to be measureable with even an optical CCD. The best solution for optimized performance is to use an electron sensitive MCP coupled to an optical CCD.

This work was performed under the auspices of the U.S. Department of Energy by the University of California, Lawrence Livermore National Laboratory under Contract No. W-7405-Eng-48. 